\title{
Increased Biosynthesis of Glycosphingolipids in Congenital Disorder of Glycosylation Ia (CDG-Ia) Fibroblasts
}

\author{
GIUSY SALA, THIERRY DUPRÉ, NATHALIE SETA, PATRICE CODOGNO, AND \\ RICCARDO GHIDONI \\ San Paolo University Hospital, University of Milan [G.S., R.G.], Milano, Italy; INSERM U410, \\ Neuroendocrinologie et Biologie Cellulaire Digestives, Faculté de Médicine Xavier Bichat [G.S.], Paris, \\ France; Réseau de Recherche sur les CDG. (INSERM/AFM) 4MR29F, Laboratoire de Biochimie A, \\ Hôpital Xavier Bichat [T.D., N.S.], Paris, France; INSERM U504, [T.D., P.C.], Villejuif, France
}

ABSTRACT

\begin{abstract}
Congenital disorder of glycosylation Ia (CDG-Ia) is an autosomal recessive disease, characterized by the impaired biosynthesis of the $\mathrm{N}$-linked oligosaccharide chains of proteins due to a deficiency of phosphomannomutase (PMM), the enzyme converting mannose6-phosphate into mannose-1-phosphate. We investigated the consequences of the altered N-linked glycoprotein (GP) biosynthesis on the quantity and quality of glycosphingolipids (GSLs) in fibroblasts of CDG-Ia patients. First, we found that CDG-Ia fibroblasts contain an increased amount of total GSLs when compared with normal fibroblasts. Further, we assessed by metabolic labeling of CDG-Ia fibroblasts with radioactive sugar precursors, including galactose and $\mathrm{N}$-acetylmannosamine, that a diminished biosynthesis of cellular GPs is antagonized by an increased biosynthesis of GSLs. An increased GSL biosynthesis was also observed by means of radiolabeled lipid precursors including sphingosine and lactosylceramide. Notably, also the degradation of GLSs is slowed down in CDG-Ia fibroblasts. Finally, when we labeled normal human fibroblasts and $\mathrm{CHO}$ cells with radioactive galactose in the presence and absence of deoxymannojirimycin (dMM), an inhibitor of N-glycan processing, we found that this cellular model mimics what occurs in CDG-Ia fibroblasts. Since an inverse relationship between GP expression and GSL content does exist, we assume that increased glycosphingolipid biosynthesis is secondary to protein hypoglycosylation. Al-
\end{abstract}

together, our data suggest that the cell metabolic machinery may be able to partially re-equilibrate protein hypoglycosylation with increased biosynthesis of glycosphingolipids, possibly to preserve the overall physico-chemical equilibrium of the outer layer of the plasma membrane. (Pediatr Res 52: 645-651, 2002)

\section{Abbreviations}

CDG-Ia, congenital disorder of glycosylation Ia;

Cer, ceramide

CHO, Chinese hamster ovary

dMM, deoxymannojirimycin

GC, glucosylceramide

GP, glycoprotein

GSL, glycosphingolipid

LC, lactosylceramide

Gb3, globotrihexosylceramide

Gb4, globotetrahexosylceramide

SM, sphingomyelin;

PDMP, threo-1-phenyl-2-decanoylamino-3-morpholino-1-

propanol

PMI, phosphomannoisomerase

PMM, phosphomannomutase

SD, standard deviation
Glycoproteins (GPs) and glycosphingolipids (GSLs) represent two major classes of glycoconjugates present in mammalian cells. The glycosylation of both classes of molecules

Received October 15, 2001; Accepted May 22, 2002.

Correspondence: Riccardo Ghidoni, San Paolo University Hospital, University of Milan, Via A. Di Rudini’, 8, I-20142 Milano, Italy; e-mail: Riccardo.Ghidoni@unimi.it

This work was supported by institutional funding from the Institut National de la Santé et de la Recherche Médicale (INSERM), an INSERM-AFM research network grant (4MR29F) "Réseau de Recherche sur les CDG" and by grants from the Association Vaincre les Maladies Lysosomales.

R.G. was a visiting professor, supported by a Poste Orange INSERM; G.S. was recipient of a Fellowship from the University of Milan.

DOI: 10.1203/01.PDR.0000032383.67029.F7 shares some common features. Noteworthy, 1) the subcellular distribution of GP and GSL glycosylating enzymes is similar, with the Golgi apparatus being the compartment where the majority of glycosylations occurs (1-3);2) some of the enzymes which glycosylate proteins and lipids are the same, e.g. those responsible for blood group antigenicity $(4,5) ; 3)$ the precursor pool of nucleotide-sugars of both GP and GSL glycosylation within the Golgi lumen is indistinguishable (6); 4) the GP and GSL transport through the Golgi complex displays biochemical and kinetical similarities (7); 5) a relevant portion of neosynthesized GPs and the totality of GSLs are delivered from the Golgi to the plasma membrane, the final site 
of localization. The distribution of glycoconjugates, GPs and GSLs, on the plasma membrane of mammalian cells contributes to the organization of the glycocalyx, the complex of the oligosaccharide chains protruding from the membrane toward the extracellular environment. The glycocalyx maintains the electrical, physical and chemical equilibrium of the plasma membrane and is involved in prominent cell functions, including cell-cell interaction, adhesion, signal transduction.

In 1980, Jaeken et al. (8) described a hereditary multisystem disorder, biochemically characterized by defect in the biosynthesis of the oligosaccharide moiety of GPs and associated with psychomotor and mental retardation and abnormalities in blood coagulation. This disorder, formerly called carbohydrate deficient glycoprotein syndrome, CDGS, has been recently renamed congenital disorder of glycosylation, CDG (9) and represents the first genetic disease affecting the biosynthesis of N-linked GP glycochains. Several CDG forms are known and the enzymatic deficiencies have been already identified (reviewed in Ref 10). The most common form of the disease is called CDG-Ia. In CDG-Ia patients the primary defect is a deficiency of phosphomannomutase (PMM), the enzyme converting mannose-6-phosphate into mannose-1-phosphate (10). The gene involved, PMM2, has been cloned $(11,12)$.

We set out to use CDG-Ia as a model system to investigate the consequences of an altered GP biosynthesis on the content of GSLs. More specifically, we tested whether there is a relationship between lipid and protein glycosylation, since this may represents a compensatory mechanism, capable to preserve the overall physico-chemical equilibrium of the outer layer of the cell plasma membrane.

Here, we report that CDG-Ia fibroblasts contain a higher level of GSLs, compared with normal fibroblasts. In addition, by metabolic studies with radioactive precursors, we observed a diminished biosynthesis of cellular GPs, concomitant with an increased biosynthesis of GSLs. Similarly, increased GSL biosynthesis was observed with radioactive lipid precursors, together with diminished GSL degradation. Finally, we used normal human fibroblasts and $\mathrm{CHO}$ cells labeled with radioactive galactose in the presence and absence of $\mathrm{dMM}$, an inhibitor of N-glycan maturation (13) and found that there is an inverse relationship between GP expression and GSL content.

\section{METHODS}

Cells and cell cultures. Human fibroblasts were obtained from upper arm skin biopsies of five PMM deficient CDG-Ia patients (three males, 2, 3, and 6 years old and two females, 4 and 6 years old) and of five sex- and age-matched controls, with disorders known not to express the transferrin abnormality of any form of CDG-Ia. All CDG-Ia patients displayed the major abnormalities typical of this disease, namely growth and mental retardation, hepatopathy, cerebellar hypoplasia/atrophy and ataxia, polyneuropathy, and dysmorphic features and displayed serum transferrin multiple band pattern $(14,15)$. CDG-Ia fibroblasts were characterized by defective mannose uptake and defective PMM activity $(<1.2 \mathrm{nmoles} / \mathrm{min} / \mathrm{mg}$ protein). Table 1 summarizes the mutations involved in PMM2 gene, when published $(16,17)$, and both phosphomannomutase (PMM) and phosphomannoisomerase (PMI) activities of CDG-Ia patient fibroblasts.

Fibroblast cultures, established from skin punch biopsies, were grown in DMEM, supplemented with 10\% FCS and serially passaged by trypsinization. Cell labeling was performed on confluent cells. All the experiments were performed at a PDL (population doubling levels) value ranging from 7 to 15 , starting from the first in vitro passage. Chinese Hamster Ovary (CHO) cells were cultured in Ham's F12 medium, supplemented with $10 \%$ FCS and serially passaged by trypsinization.

Radiolabeled precursors and metabolic labeling. D-[1$\left.{ }^{14} \mathrm{C}\right]$ Galactose (specific radioactivity: $58 \mathrm{mCi} / \mathrm{mmol}$ ) and $\mathrm{N}$-acetyl-[6- $\left.{ }^{3} \mathrm{H}\right]$ mannosamine (specific radioactivity: $39.1 \mathrm{Ci} /$ mmol) were from Amersham (Cologmo Monzese, Italy) and NEN (Boston, MA, U.S.A.), respectively. $\left[3-{ }^{3} \mathrm{H}\right]$ Sphingosine (specific radioactivity: $1.25 \mathrm{Ci} / \mathrm{mmol}),\left(\left[6-{ }^{3} \mathrm{H}\right]\right.$ gal)lactosylceramide (specific radioactivity: $0.95 \mathrm{Ci} / \mathrm{mmol}$ ) and ([3$\left.{ }^{3} \mathrm{H}\right] \mathrm{sph}$ )lactosylceramide (specific radioactivity: $1.05 \mathrm{Ci} / \mathrm{mmol}$ ) (20) were prepared in our laboratory $(18-20) \cdot 10^{5}$ cells were pulse-labeled in $1 \mathrm{~mL}$ serum-free medium, at final concentration of $1 \mu \mathrm{Ci} / \mathrm{mL}$ for galactose and sphingosine and $5 \mu \mathrm{Ci} / \mathrm{mL}$ for lactosylceramides and $\mathrm{N}$-acetylmannosamine. A pulse protocol (48 h) was used for sphingosine, lactosylceramides and $\mathrm{N}$-acetylmannosamine labeling. A pulse-chase protocol $(6 \mathrm{~h}$ pulse, $12 \mathrm{~h}$ chase) was used for galactose labeling. The chase period was initiated by substituting the pulse medium with 1 $\mathrm{mL}$ serum-free medium. In all cases, at the end of the incubation, cells were rinsed three times with ice-cold saline, harvested with a rubber policeman, pelleted, frozen and stored at $-20^{\circ} \mathrm{C}$, before lipid extraction or protein determination (21). In the case of lactosylceramide, cell labeling was also stopped after the pulse period $(6 \mathrm{~h})$, and cell treated as before, to measure the initial radioactivity incorporation.

$\boldsymbol{d M M}$ treatment. Normal human fibroblasts and $\mathrm{CHO}$ cells were pretreated with $1 \mathrm{mM} d \mathrm{dM}$ for $48 \mathrm{~h}$ followed by $6 \mathrm{~h}$

Table 1. Mutations involved in PMM2 gene and enzyme activities of the CDG-Ia patient fibroblasts used in this study

\begin{tabular}{|c|c|c|c|c|c|}
\hline Patient & PMM & PMI & Mutation 1 & Mutation 2 & Reference \\
\hline 1 & $<0.5$ & 14 & L32R & nd & np \\
\hline 2 & 1.0 & 20.3 & C241S & F157S & 16 \\
\hline 3 & $<0.5$ & 24.3 & $\mathrm{~T} 266 \mathrm{~S}$ & $\mathrm{R} 141 \mathrm{H}$ & 17 \\
\hline 5 & 1.2 & 21.7 & nd & nd & n.p \\
\hline
\end{tabular}

PMM, phosphomannomutase; PMI, phosphomannoisomerase; nd, not determined; np, not published. PMM and PMI activities are expressed as nmoles/min/mg protein. Control fibroblasts had normal PMM (ranging from 2.7 to $4.3 \mathrm{nmoles} / \mathrm{min} / \mathrm{mg}$ protein) and PMI (ranging from 18.3 to $24.3 \mathrm{nmoles} / \mathrm{min} / \mathrm{mg}$ protein) activities. 
incubation with both $1 \mathrm{mM} d \mathrm{dM}$ and $1 \mu \mathrm{Ci} / \mathrm{mL} \mathrm{D}-[1-$ ${ }^{14} \mathrm{C}$ ]galactose and finally $12 \mathrm{~h}$ incubation with regular medium.

PDMP treatment. Normal human skin fibroblasts were pretreated with $40 \mu \mathrm{M}$ PDMP for $24 \mathrm{~h}$ followed by a $6 \mathrm{~h}$ incubation with both $40 \mu \mathrm{M}$ PDMP and $1 \mu \mathrm{Ci} / \mathrm{mL}$ D-[1${ }^{14} \mathrm{C}$ ]galactose and finally $12 \mathrm{~h}$ incubation with regular medium. Optimal PDMP concentration was determined in preliminary experiments to minimize cytotoxicity.

Lipid extraction and analysis. To determine the endogenous GSLs content, lipids were extracted by the tetrahydrofuran/ phosphate buffer method (22), starting from $4 \times 75 \mathrm{~cm}^{2}$ dishes, for each sample. The organic phase, containing neutral GSLs, was analyzed by HPTLC, using chloroform/methanol/formic $\mathrm{acid} /$ water, 65/25/8.9/1.1, by vol, as the eluting solvent system. Visualization was performed with a $10 \% \mathrm{CuSO}_{4} / 8 \%$ phosphoric acid aqueous spraying solution. The aqueous phase, containing gangliosides, was analyzed by HPTLC, using chloroform/methanol $/ 0.2 \%$ aqueous $\mathrm{CaCl}_{2}, 6 / 4 / 1$, by vol, as the eluting solvent system. Visualization was performed with a p-dimethylaminobenzaldehyde spray reagent (23). Quantification of the endogenous GSL pattern was performed by scanning densitometry, using commercial standards as reference compounds. To analyze radioactive GSLs and ceramide, lipids were extracted by the chloroform/methanol/pyridine/water method (24). HPTLC separation was performed using chloroform/methanol/water, 60/35/7, by vol, as the eluting solvent system, except in sphingosine labeling experiments, where a chloroform/methanol/formic acid/water, 65/25/6/4, by vol, solvent system was used, to separate sphingomyelin and GM3. Radioactive lipids were visualized by fluorography and identified by comparison with radiolabeled standards available in our laboratory or with commercial nonradioactive standards. The spots corresponding to known lipids were scraped from the plate and counted by liquid scintillation.

Radioactivity incorporation into cell protein. Cell pellets were delipidized with chloroform/methanol/water, 10/10/3, by vol. The residue after $900 \times g$ centrifugation, $15 \mathrm{~min}$, contain- ing radiolabeled proteins, was dissolved in $200 \mu \mathrm{L}$ soluene at $60^{\circ} \mathrm{C}$ for $1 \mathrm{~h}$ and counted by liquid scintillation.

\section{RESULTS}

Endogenous GSLs content in CDG-Ia fibroblasts versus normal fibroblasts. To investigate the consequences of an altered GP biosynthesis on the content of GSLs, we tested whether there is a relationship between lipid and protein glycosylation, by measuring the total endogenous GSL content in CDG-Ia fibroblasts versus normal fibroblasts. As reported in Table 2, we established that the qualitative GSL pattern, including the neutral GSLs GC, LC, Gb3, Gb4, and the gangliosides GM3, GM2, GD3, GM1, and GD1a (Gangliosides are designated according to Svennerholm L (1964), J Lipid Res, 5, 145-155.), was identical between CDG-Ia and normal fibroblasts. In contrast, the quantitative composition between normal and CDG-Ia fibroblast was quite different, even in the presence of identical protein and DNA content. More specifically, the content of some species of GSLs (Gb3, GM2, GD3, and GD1a) was significantly $(p<0.05)$ increased in CDG-Ia fibroblasts versus normal controls. GM2 and GD3 gangliosides were the most markedly increased.

Biosynthesis of glycoconjugates (GPS and GSLs) in CDG-Ia fibroblasts after labeling with sugar precursors. Next we tested whether there is a relationship between the GP and GSL glycosylation. To gain information on the de-novo glycosylation of both lipid and protein moieties, we labeled normal and CDG-Ia fibroblasts with $\left[{ }^{14} \mathrm{C}\right]$ galactose, and found: 1) similar qualitative patterns for radioactive GSLs and gangliosides in CDG-Ia and normal fibroblasts (Fig. 1A);2) an increase of approximately 19\% radioactivity incorporation in CDG-Ia fibroblasts (Fig. 1B) [155,600 \pm 19,980 (mean value \pm SD); 125,900-179,800 (range); 153,600 (median) dpm/mg protein] versus control fibroblasts $[131,100 \pm 7,310$ (mean value $\pm \mathrm{SD}$ ); 120,100-139,500 (range); 133,100 (median) $\mathrm{dpm} / \mathrm{mg}$ protein, $p<0.05$ ]; 3) a distribution of this increased

Table 2. Neutral GSL and ganglioside endogenous pattern of CDG-Ia and control fibroblasts

\begin{tabular}{|c|c|c|c|c|c|c|c|c|}
\hline $\begin{array}{c}\text { Cell } \\
\text { population }\end{array}$ & $\mathrm{GC}$ & $\mathrm{LC}$ & Gb3 & $\mathrm{Gb} 4$ & GM3 & GM2 & GD3 & GD1a \\
\hline \multicolumn{9}{|l|}{ Controls } \\
\hline 1 & 2.45 & 3.45 & 4.70 & 1.86 & 2.36 & 0.05 & 0.52 & 0.11 \\
\hline 3 & 2.59 & 3.20 & 4.30 & 1.98 & 2.12 & 0.03 & 0.32 & 0.12 \\
\hline 4 & 2.38 & 3.25 & 4.98 & 1.97 & 2.54 & 0.08 & 0.35 & 0.09 \\
\hline 5 & 2.64 & 3.45 & 4.25 & 1.76 & 2.24 & 0.04 & 0.43 & 0.10 \\
\hline \multicolumn{9}{|l|}{ CDG-la } \\
\hline 1 & 2.70 & 3.72 & 4.91 & 2.21 & 2.57 & 0.09 & 0.79 & 0.11 \\
\hline 2 & 2.57 & 3.65 & 5.32 & 1.90 & 2.35 & 0.11 & 0.71 & 0.13 \\
\hline 3 & 2.81 & 3.90 & 5.11 & 1.79 & 2.64 & 0.07 & 0.59 & 0.14 \\
\hline 4 & 2.34 & 3.54 & 5.09 & 2.15 & 2.43 & 0.07 & 0.62 & 0.11 \\
\hline 5 & 2.89 & 3.59 & 4.78 & 2.19 & 2.31 & 0.13 & 0.69 & 0.12 \\
\hline
\end{tabular}

Neutral GSLs and gangliosides were separated by HPTLC and quantified by scanning densitometry, using known amounts of spotted commercial standard as reference. Data in the table are expressed as nmol/mg protein. ns, not significant. 
radioactivity in all lipids, the most remarkable changes concerning GC, Gb3, GD3 and GM2 (Fig. 1C); 4) a reduced incorporation of $\left[{ }^{14} \mathrm{C}\right]$ galactose into the cell protein glycochains in CDG-Ia fibroblasts (Fig. 1B). This accounted for about $28 \%$ [242,300 $\pm 27,410$ (mean value $\pm \mathrm{SD}$ ); 201,000-
A

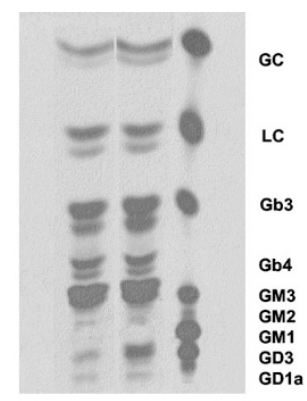

C CDG-la st

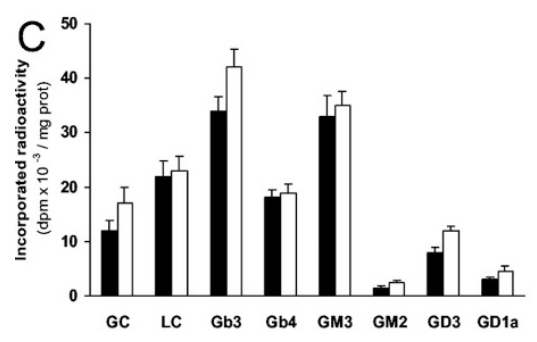

B
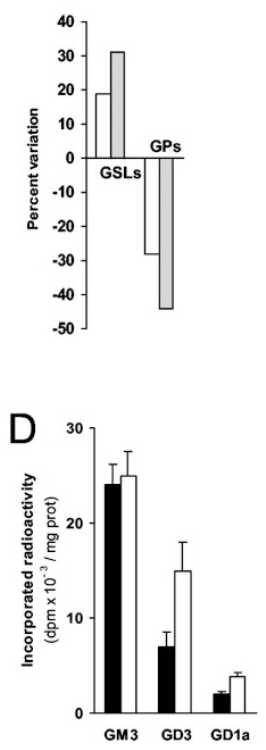

Figure 1. Biosynthesis of glycoconjugates (GPs and GSLs) in CDG-Ia fibroblasts after labeling with sugar precursors. (A) TLC separation of radioactive GSLs in control and CDG-Ia fibroblasts, after $\left[{ }^{14} \mathrm{C}\right]$ galactose labeling. Radioactive GSLs, after extraction, were separated by HPTLC and visualized by fluorography. Eluting solvent system: chloroform/methanol/water, 60/35/7, by vol. Lane C: GSLs of fibroblasts from control 4; lane CDG-Ia: GSLs of fibroblasts from patient 2; lane St: reference radioactive GSLs. $(B)$ Percent variation of radioactivity incorporation into GPs and GSLs after metabolic labeling with sugar precursors in CDG-Ia, referred to normal control fibroblasts. Results are the mean values of five experiments. Empty bars, $\left[{ }^{14} \mathrm{C}\right]$ galactose labeling; full bars: $\mathrm{N}$-acetyl- $\left[{ }^{3} \mathrm{H}\right]$ mannosamine labeling. $(C)$ Quantitative determination of radioactivity incorporation into individual GLSs after $\left[{ }^{14} \mathrm{C}\right]$ galactose labeling. After visualization by fluorography, individual spots were scraped off the plate and counted by liquid scintillation. Results are the mean values $\pm \mathrm{SD}$ of individual experiments of five control and five CDG-Ia fibroblasts. Full bars: control fibroblasts; empty bars: CDG-Ia fibroblasts. Further statistical data (all expressed as dpm $\times 10^{-3 / m g}$ protein): Control fibroblasts, range values: GC, 10.2-14.4; LC, 17.3-24.3; Gb3, 30.3-37.2; Gb4, 15.1-19.2; GM3, 28.3-36.9; GM2, 1.2-1.8; GD3, 7.1-10.3; GD1a, 2.0-4.7; medians: GC, 12.1; LC, 22.0; Gb3, 32.9; Gb4, 16.8; GM3, 34.5; GM2, 1.6; GD3, 8.1; GD1a, 3.1. CDG-Ia fibroblasts, range values: GC, 13.4-21.4; LC, 18.2-25.2; Gb3, 38.4-46.8; Gb4, 16.3-20.1; GM3, 30.4-37.3; GM2, 2.3-2.9; GD3, 10.3-13.5; GD1a, 3.2-5.6; medians: GC, 16.9; LC, 22.8; Gb3, 42.6; Gb4, 18.0; GM3, 35.7; GM2, 2.4; GD3, 12.0; GD1a, 4.1. Significance, CDG-Ia vs controls: GC, Gb3, GD3 and GM2 $p<$ 0.05 ; others: not significant. $(D)$ Quantitative determination of radioactivity incorporation into individual GLSs after $\mathrm{N}$-acetyl- $\left[{ }^{3} \mathrm{H}\right]$ mannosamine labeling. Radioactive gangliosides, after extraction, were separated by HPTLC and visualized by fluorography. Individual spots were scraped off the plate and counted by liquid scintillation. Results are the mean values $\pm \mathrm{SD}$ of individual experiments of five control and five CDG-Ia fibroblasts. Full bars: control fibroblasts; empty bars: CDG-Ia fibroblasts. Further statistical data (all expressed as $\mathrm{dpm} \times 10^{-3} / \mathrm{mg}$ protein): Control fibroblasts, range values: GM3, 21.2-27.0; GD3, 5.0-8.9; GD1a, 1.5-2.9; medians: GM3, 24.1; GD3, 7.8; GD1a, 2.1. CDG-Ia fibroblasts, range values: GM3, 22.3-27.9; GD3, 13.521.2; GD1a, 2.8-4.2; medians: GM3, 25.6; GD3, 16.1; GD1a, 3.6. Significance, CDG-Ia $v s$ controls: GD3 and GD1a, $p<0.05$; GM3, not significant.
276,800 (range); 245,700 (median) dpm/mg protein, in control fibroblasts versus $188,900 \pm 16,010$ (mean value $\pm \mathrm{SD}$ ); 165,400-204,200 (range); 188,200 (median) dpm/mg protein in CDG-Ia ones, $p<0.05$ ].

To evaluate the sialylation rates of gangliosides and GPs, we labeled cells with $\mathrm{N}$-acetylmannosamine, and observed that 1 ) the incorporated radioactivity was about $31 \%$ higher in CDG-Ia [44,200 \pm 5,280 (mean value \pm SD); 37,800-52,000 (range); 43,100 (median) dpm/mg protein] than in control $[33,700 \pm 4,070$ (mean value $\pm \mathrm{SD}$ ); 28,300-39,000 (range); 34,600 (median) dpm/mg protein, $p<0.05$ ] specimens, thus indicating that sialylation is significantly augmented (Fig. $1 B$ ); 2) significant changes concern the disialylated gangliosides GD3 and GD1a (Fig. 1D), that are approximately 2-fold more radioactive in CDG-Ia with respect to control cells; 3) radioactivity incorporation into cell GPs was dramatically impaired $(45 \%)$ [168,100 $\pm 21,350$ (mean value $\pm \mathrm{SD}$ ); 138,600197,200 (range); 165,900 (median) dpm/mg protein in CDG-Ia versus 299,800 \pm 36,550 (mean value $\pm \mathrm{SD}$ ); 246,900340,000 (range); 296,900 (median) dpm/mg protein in controls, $p<0.05$ ] (Fig. 1B).

In conclusion, these data show that there is an inverse relationship between GP and GSL biosynthesis in CDG-Ia fibroblasts.

Biosynthesis of glycosylated and nonglycosylated sphingolipids in CDG-Ia fibroblasts after labeling with lipid precursors. To test whether the previous data could be related to the rate of incorporation of radiolabeled sugars into the nucleotidesugar pool that may be different between CDG-Ia and normal fibroblasts (25), we metabolically labeled cells with nonsugar precursors, including $\left(\left[{ }^{3} \mathrm{H}\right]\right.$ gal $)$ lactosylceramide and $\left[{ }^{3} \mathrm{H}\right]$ sphingosine.

Exogenous lactosylceramide can be used in vivo and in intact cells as a substrate for the biosynthesis of more glycosylated GSLs (26). As can be seen in Fig. 2A, CDG-Ia fibroblasts incorporated $15-34 \%$ more radioactivity in $\mathrm{Gb} 3, \mathrm{~Gb} 4$, and GM3, when compared with normal control fibroblasts.

To gain information on the de-novo biosynthesis of the sphingolipid backbone in CDG-Ia fibroblasts, we labeled these cells with $\left[{ }^{3} \mathrm{H}\right]$ sphingosine and observed that the radioactivity incorporation in Gb3, GD3 and GD1a was remarkably increased, over the normal fibroblasts (Fig. 2B). Noteworthy, the incorporation of radioactivity into nonglycosylated sphingolipids, ceramide and SM, showed that ceramide biosynthesis remained unmodified, whereas SM biosynthesis was slightly but significantly reduced (Fig. 2C).

Altogether these data suggest that the nucleotide-sugar pool, when not used for GP biosynthesis, may be available to lipid glycosylation.

GSL degradation in CDG-Ia fibroblasts after labeling with ([3H]sph)lactosylceramide. To assess on the lysosomal GSLs degradation rates in CDG-Ia fibroblasts, we labeled cells with $\left(\left[{ }^{3} \mathrm{H}\right] \mathrm{sph}\right)$ lactosylceramide. We used this molecule, since the labeling on the sphingoid base is conserved in degradation products originated from lactosylceramide itself (19). We detected a consistent impairment of glycohydrolases-assisted deglycosylation in CDG-Ia fibroblasts. Particularly, glucosylceramide and ceramide were $25 \%$ to $30 \%$ lower than in normal 
A

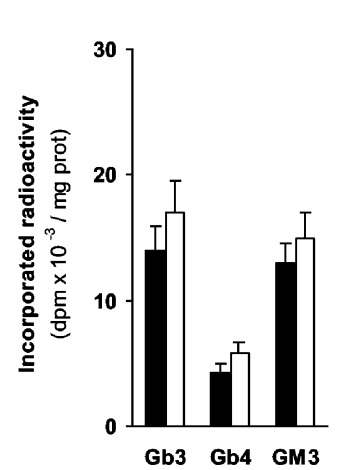

C

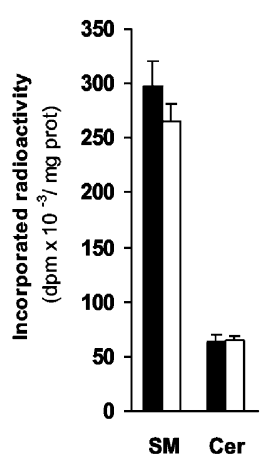

B

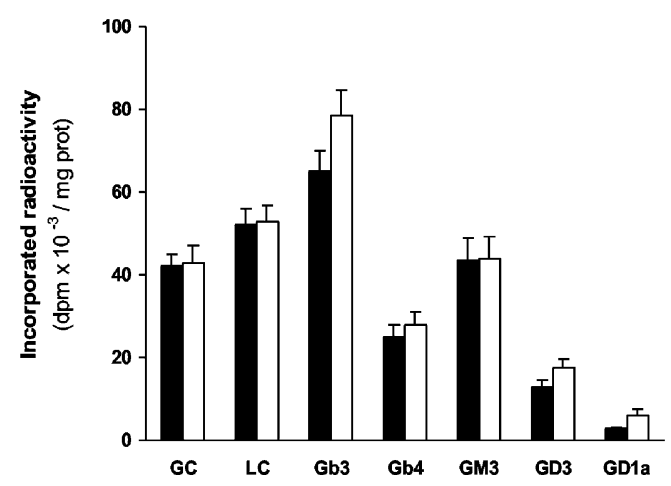

Figure 2. Biosynthesis of glycosylated and nonglycosylated sphingolipid in CDG-Ia fibroblasts after labeling with lipid precursors. (A) Quantitative determination of radioactivity incorporation into individual radioactive GSLs in control and CDG-Ia fibroblasts, after $\left(\left[{ }^{3} \mathrm{H}\right]\right.$ gal)lactosylceramide labeling. Radioactive GSLs, after extraction, were separated by HPTLC and visualized by fluorography. Individual spots were scraped off the plate and counted by liquid scintillation. Results are the mean values \pm SD of individual experiments of five control and five CDG-Ia fibroblasts. Full bars: control fibroblasts; empty bars: CDG-Ia fibroblasts. Further statistical data (all expressed as dpm $\times 10$

$-3 / \mathrm{mg}$ protein): Control fibroblasts, range values: $\mathrm{Gb3}, 10.6-15.7$; Gb4, $3.6-$ 5.1; GM3, 11.6-17.0; medians: Gb3, 13.5; Gb4, 4.1; GM3, 13.5. CDG-Ia fibroblasts, range values: $\mathrm{Gb} 3,14.2-19.3$; Gb4, 4.9-7.2; GM3, 12.6-18.1; medians: Gb3, 17.9; Gb4, 6.1; GM3, 14.7. Significance, CDG-Ia vs controls: $\mathrm{Gb} 3$ and $\mathrm{Gb} 4, p<0.05$. GM3, not significant. $(B)$ Quantitative determination of radioactivity incorporation in individual radioactive GSLs in control and CDG-Ia fibroblasts after $\left[{ }^{3} \mathrm{H}\right]$ sphingosine labeling. Radioactive GSLs, after extraction, were separated by HPTLC and visualized by fluorography. Individual spots were scraped off the plate and counted by liquid scintillation. Results are the mean values $\pm \mathrm{SD}$ of individual experiments of five control and five CDG-Ia fibroblasts. Full bars: control fibroblasts; empty bars: CDG-Ia fibroblasts. Further statistical data (all expressed as dpm $\times 10^{-3} / \mathrm{mg}$ protein): Control fibroblasts, range values: GC, 35.4-44.6; LC, 47.2-56.1; Gb3, 57.2-69.8; Gb4, 20.4-28.1; GM3, 36.1-49.2; GD3, 12.1-16.9; GD1a, 3.1-3.9; medians: GC, 42.5; LC, 51.9; Gb3, 64.3, Gb4, 23.5; GM3, 42.2; GD3, 13.5; GD1a, 3.3. CDG-Ia fibroblasts, range values: GC, 38.1-47.7; LC, 49.2-56.0; Gb3, 70.4-86.2; Gb4, 22.9-31.1; GM3, 36.5-50.3; GD3, $14.2-$ 19.3; GD1a, 4.2-7.5: medians: GC, 44.6; LC, 53.9; Gb3, 79.2; Gb4, 28.2; GM3, 44.1; GD3, 13.5; GD1a, 6.4. Significance, CDG-Ia vs controls: Gb3, GD3 and GD1a $p<0.05$; others, not significant. GM2 was not distinguishable from GM3. (C) Quantitative determination of radioactivity incorporation in individual radioactive sphingomyelin and ceramide in control and CDG-Ia fibroblasts after $\left[{ }^{3} \mathrm{H}\right]$ sphingosine labeling. Radioactive sphingolipids, after extraction, were separated by HPTLC and visualized by fluorography. Individual spots were scraped off the plate and counted by liquid scintillation. Results are the mean values $\pm \mathrm{SD}$ of individual experiments of five control and five CDG-Ia fibroblasts. Full bars: control fibroblasts; empty bars: CDG-Ia fibroblasts. Further statistical data (all expressed as dpm $\times 10^{-3} / \mathrm{mg}$ protein): Control fibroblasts, range values: SM, 281.3-320.0; Cer, 52.3-71.9; medians, SM, 289.4; Cer, 64.6. CDG-Ia fibroblasts, range values: SM, 222,3270.3; Cer, 54.4-71.1; medians, SM, 243.3; Cer, 66.6. Significance, CDG-Ia $v s$ controls: $\mathrm{SM} p<0.05$; Cer, not significant. fibroblasts (Fig. 3), even in the presence of similar incorporation of radioactivity after the pulse period between control $[412,700 \pm 47,660$ (mean value $\pm \mathrm{SD}$ ); 356,800-487,700 (range); 409,600 (median) dpm/mg protein] and CDG-Ia $[420,800 \pm 49,990$ (mean value $\pm \mathrm{SD}$ ); $347,100-486,600$ (range); 426,000 (median) dpm/mg protein] fibroblasts. We conclude that the GSLs catabolism is decreased in CDG-Ia cells. This is supported by the finding that CDG-Ia fibroblasts displayed 30\% less SM than normal controls, since the biosynthesis of SM occurs via a recycling route of the free sphingoid base released in the lysosomes (19).

Biosynthesis of glycoconjugates (GPS and GLS) in normal human fibroblasts and CHO cells after dMM and PDMP treatment. To test whether sphingolipid glycosylation is secondary to protein hypoglycosylation, we labeled normal human fibroblasts and $\mathrm{CHO}$ cells with radioactive galactose in the presence and absence of deoximannojirimycin (dMM), an inhibitor of N-glycan processing. As shown in Fig. 4, we observed a 33\% decrease of GP biosynthesis and a concomitant $25 \%$ increase of GSL biosynthesis in dMM treated versus untreated normal human fibroblasts. The GSL molecular species undergoing significant increase were GC (35\%), Gb3 $(18 \%)$, GM2 (57\%), and GD3 (23\%). Differences in other species were not significant.

To assess whether a reciprocal effect does occur, normal human fibroblasts were also labeled with radioactive galactose in the presence and absence of PDMP, an inhibitor of glucosylceramide synthase, the first enzyme in the multi-step glycosylation of GSLs. Concurrent to a 30\% inhibition of sphingolipid glycosylation, no significant increase of protein glycosylation

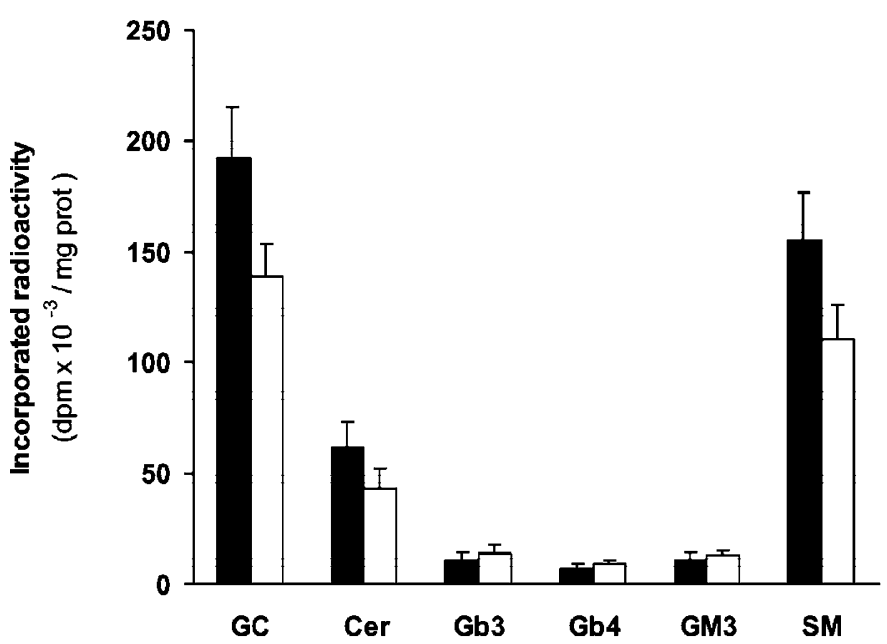

Figure 3. GSL degradation in CDG-Ia fibroblasts after labeling with $\left(\left[{ }^{3} \mathrm{H}\right] \mathrm{sph}\right)$ lactosylceramide. Radioactive sphingolipids, after extraction, were separated by HPTLC and visualized by fluorography. Individual spots were scraped off the plate and counted by liquid scintillation. Results are the mean values $\pm \mathrm{SD}$ of individual experiments of five control and five CDG-Ia fibroblasts. Full bars: control fibroblasts; empty bars: CDG-Ia fibroblasts. Further statistical data (all expressed as dpm $\times 10^{-3} / \mathrm{mg}$ protein): Control fibroblasts, range values: GC, 170.3-226.3; Cer, 50.4-75.9; Gb3, 6.2-17.2; Gb4, 4.8-11.7; GM3, 5.5-15.6; SM, 140.3-185.8; medians: GC; 194.4; Cer, 59.9; Gb3, 11.4; Gb4, 6.0; GM3, 9.9; SM, 162.2. CDG-Ia fibroblasts, range values: GC, 128.3-168.2; Cer, 33.4-57.8; Gb3, 9.2-19.2; Gb4, 5.7-12.7; GM3, 8.2-15.6; SM, 103.5-140.6; medians: GC, 145.4; Cer, 41.7; Gb3, 13.0; Gb4, 8.5; GM3, 12.7; SM, 119.9. Significance, CDG-Ia vs controls: GC, Cer, $\mathrm{SM}, p<0.05$; others, not significant. 


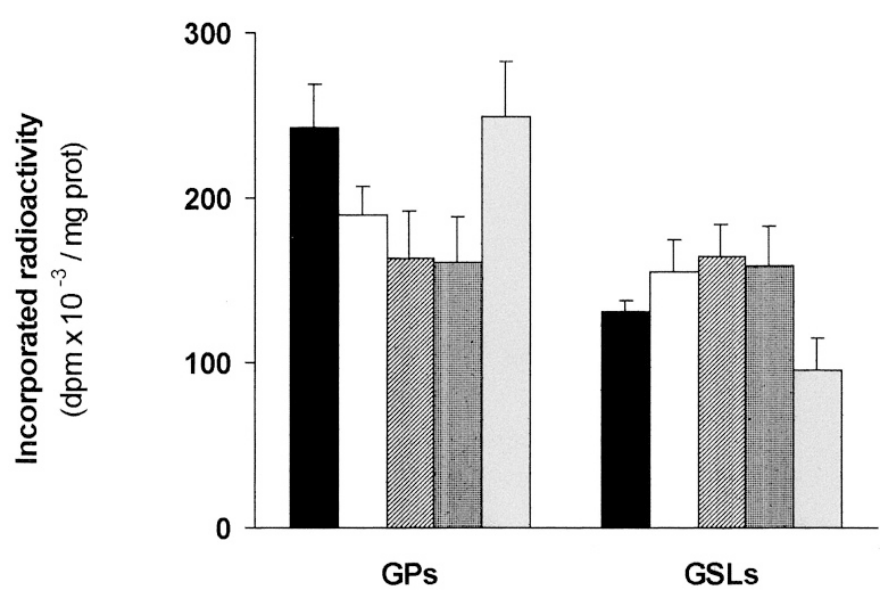

Figure 4. Effect of dMM and PDMP treatments on radioactivity incorporation into GSLs and GPs after $\left[{ }^{14} \mathrm{C}\right]$ galactose labeling. Incorporated radioactivity was measured by liquid scintillation. Results are the mean values \pm SD of five individual experiments. $\square$ untreated normal human fibroblasts; $\square$ CDG-Ia fibroblasts; 四; dMM-treated normal human fibroblasts; 眻 dMMtreated $\mathrm{CHO}$ cells; $\square$ PDMP-treated normal human fibroblasts. Further statistical data (all expressed as $\mathrm{dpm} \times 10^{-3} / \mathrm{mg}$ protein). Untreated normal human fibroblasts and CDG-Ia fibroblasts, see text. dMM-treated normal human fibroblasts; GPs, range values: 139.5-206.8; median: 169.8; GSLs, range values, 136.3-180.8; median, 167.7. dMM-treated CHO cells; GPs, range values: 140.1-209.1; median: 162.2; GSLs, range values, 140.2-194.2; median, 167.0. PDMP-treated normal human fibroblasts: GPs, range values: 216.1-301.8; median: 239.5; GSLs, range values, 72.5-120.5; median: 93.2. Significance: GPs, CDG-Ia, dMM-treated normal human fibroblasts and dMM-treated CHO cells $v s$ untreated normal human fibroblasts, $p<0.05$, PDMP-treated normal human fibroblasts $v s$ untreated normal human fibroblasts, not significant; GSLs, CDG-Ia and all treated fibroblasts $v s$ untreated normal human fibroblasts: $p<0.05$.

occurs in PDMP treated fibroblasts (Fig. 4). In conclusion, these data suggest that increase of GSLs is secondary to protein hypoglycosylation and that the reciprocal phenomenon does not occur.

\section{DISCUSSION}

The most relevant finding of the present work is an increased GSL content in CDG-Ia fibroblasts, compared with normal matched fibroblasts, apparent from the quantitation of endogenous GSLs, under strictly controlled experimental conditions. This was necessary since the endogenous pattern of GSLs and gangliosides in human fibroblasts is dramatically dependent on various factors, including cell density (27), tissue of origin (28) and individual variability (29). To minimize any bias due to these factors, we performed all the experiments with fibroblasts from patients matched with normal individuals of the same age of the patient ones and at the same passage in culture. We found that the endogenous profile of GSLs was in agreement with previously published profiles (29-31) and included the most represented species GC, LC, Gb3, Gb4, GM3, GD3 and the less abundant species GD1a and GM2. The increase of total GSL content in CDG-Ia patients is, to our knowledge, the first evidence of a compensatory mechanism aimed to re-equilibrate disturbed protein glycosylation by increased GSL biosynthesis.

Our supposition was corroborated by a series of labeling studies. We showed that increased GLSs content is not conse- quent to a single step, as demonstrated by lack of specificity of incorporation, when different (sugar or lipid) radioactive precursors were used.

Most notably, in parallel with GSL increase, there was a marked reduction of protein glycosylation in CDG-Ia fibroblasts versus control cells. This was evident with galactose labeling and even more evident with $\mathrm{N}$-acetylmannosamine labeling. This result demonstrates that nonsecreted GP biosynthesis is affected in CDG-Ia patients, in addition to the known secreted GP biosynthesis defect (8). The impairment of protein glycosylation in general, and sialylation in particular, corroborates a recent observation (32), reporting a reduced surface expression of sialoglycans on B lymphocytes from CDG-Ia patients. We speculate that the combined impairment of galactose and $\mathrm{N}$-acetylmannosamine incorporation into GPs could be due to a diminished availability of core structures and/or a delayed GP transport along the secretory pathway $(33,34)$.

A diminished GP biosynthesis is concurrent with an increased incorporation of the same sugar precursors into GSLs. This demonstrates the occurrence of inverse relationship between GP and GSL contents in CDG-Ia fibroblasts. The Golgi apparatus is likely to contribute in supporting this phenomenon.

By the results obtained with lipid precursors, one attractive evidence was that SM biosynthesis is slightly depressed, thus suggesting that the ceramide precursor pool is preferentially used for glycosylating purposes, in CDG-Ia.

As far as the GSL degradation was concerned, we demonstrated that it is impaired in CDG-Ia versus control fibroblasts. This finding is in agreement with the evidence (35) that the activity of lysosomal enzymes is decreased in CDG-Ia cells.

We finally observed a higher GSL content in normal human and murine fibroblasts, treated with $\mathrm{dMM}$, an inhibitor of GP biosynthesis. The metabolic change of dMM-treated fibroblasts resembles that occurring in CDG-Ia fibroblasts, suggesting that the increase of GSLs is actually depending on protein hypoglycosylation. Since no reciprocal effect was observed on protein glycosylation after PDMP inhibition of GSL biosynthesis, we presume different regulatory mechanisms for protein and lipid glycosylation.

Altogether, this paper shows that the increased sphingolipid biosynthesis is secondary to protein hypoglycosylation in CDG-Ia fibroblasts. This may represent a cellular process devised to preserve the homeostasis of the plasma membrane constituents.

Acknowledgments. The authors thank Dr. S. Moore and Dr. N. Sacchi for critical reading of the manuscript, Dr. V. Cormier-Daire for providing CDG-Ia fibroblasts, Dr. G. Durand for useful discussion, and Dr. M. Laburthe for access to facilities during the course of the present work.

\section{REFERENCES}

1. Paulson JC, Colley KJ 1989 Glycosyltransferases. Structure, localization, and control of cell type-specific glycosylation. J Biol Chem 264:17615-17618

2. Keenan TW, Morré DJ, Basu S 1974 Concentration of glycosphingolipid glycosyltransferases in Golgi apparatus from rat liver. J Biol Chem 249:310-315

3. Trinchera M, Pirovano B, Ghidoni R 1990 Sub-Golgi distribution in rat liver of CMP-NeuAc:GM3- and CMP-NeuAc:GT1b-alpha 2-8sialyltransferases and compar- 
ison with the distribution of the other glycosyltransferase activities involved in ganglioside biosynthesis. J Biol Chem 265:18242-18247

4. Roseman S 2001 Reflections on Glycobiology J Biol Chem 276:41527-41542

5. Hakomori S-I 1999 Antigen structure and genetic basis of histo-blood groups A, B and $\mathrm{O}$ : their changes associated with human cancer. Biochim Biophys Acta 1473:247-266

6. Briles EB, Kornfeld S 1977 Isolation of wheat germ agglutinin-resistant clones of Chinese hamster ovary cells deficient in membrane sialic acid and galactose. J Biol Chem 252:1107-1116

7. Wattemberg BW 1990 Glycolipid and glycoprotein transport through the Golgi complex are similar biochemically and kinetically. Reconstitution of glycolipid transport in a cell free system. J Cell Biol 111:421-428

8. Jaeken JM, Vanderschueren-Lodeweyckx M, Casaer P, Snoeck L, Corbeel L, Eggermont E, Eeckels R 1980 Familiar psychomotor retardation with markedly fluctuating serum prolactin, FSH and GH levels, partial TBG deficiency, increased serum prolactin, FSH and GH levels, partial TBG deficiency, increased CSF protein: a new syndrome? Pediatr Res 14:179

9. Participants, "First International Workshop on CDGS", Leuven, Belgium, November 12-13, 1999, 1999 Letter to the Editor. Carbohydrate-deficient glycoprotein syndromes become congenital disorders of glycosylation: an updated nomenclature for CDG. Glycoconj J 16:669-671

10. Aebi M, Hennet T 2001 Congenital disorders of glycosylation: genetic model systems lead the way. Trends Cell Biol 11:136-141

11. Matthijs G, Schollen E, Pardon E, Veiga da Cunha M, Jaeken J, Cassiman JJ, Van Schaftingen E 1997 Mutations in PMM2, a phosphomannomutase gene on chromosome $16 \mathrm{p} 13$, in carbohydrate-deficient glycoprotein type I syndrome (Jaeken syndrome). Nat Genet 16:88-92

12. Matthijs G, Schollen E, Bjursell C, Erlandson A, Freeze H, Imtiaz F, Kjaergaard S, Martinsson T, Schwartz M, Seta N, Vuillaumier-Barrot S, Westphal V, Winchester B 2000 Mutations in PMM2 that cause congenital disorders of glycosylation, type Ia (CDG-Ia). Hum Mutation 16:386-394

13. Fuhrmann U, Bause E, Legler G, Ploegh H 1984 Novel mannosidase inhibito blocking conversion of high mannose to complex oligosaccharides. Nature 307:755768

14. Seta N, Barnier A, Hochedez F, Besnard AM, Durand G 1996 Diagnostic value of Western blotting in Carbohydrate-deficient glycoprotein syndrome. Clin Chim Acta 254:131-140

15. Dupré T, Ogier-Denis E, Moore S, Cormier-Daire V, Dehoux M, Durand G, Seta N, Codogno P 1999 Alteration of mannose transport in fibroblasts from type I carbohydrate deficient glycoprotein syndrome patients. Biochim Biophys Acta 1453:369-377

16. Drouin-Garraud V, Belgrand M, Grunewald S, Seta N, Dacher JN, Henocq A, Matthijs G, Cormier-Daire V, Frebourg T, Saugier-Veber P 2001 Neurological presentation of a congenital disorder of glycosylation CDG-Ia: implications for diagnostic and genetic counseling. Am J Med Genet 101:46-49

17. de Lonlay P, Seta N, Barrot S, Chabrol B, Drouin V, Gabriel BM, Journel H, Kretz M, Laurent J, Le Merrer M, Leroy A, Padespan D, Sarda P, Villeneuve N, Schmitz J, van Schaftingen E, Matthijs G, Jaeken J, Korner C, Munnich A, Saudubray JM, Corner-Daire V 2001 A broad spectrum of clinical presentations in congenital disorders of glycosylation Ia: a series of 26 cases J Med Genet 38:14-19

18. Ghidoni R, Houri JJ, Giuliani A, Ogier-Denis E, Parolari E, Botti S, Bauvy C, Codogno P 1996 The metabolism of sphingo(glyco)lipids is correlated with the differentiation-dependent autophagic pathway in HT-29 cells. Eur J Biochem 237:454-459

19. Trinchera M, Ghidoni R 1990 Subcellular biosynthesis and transport of gangliosides formed from exogenous lactosylceramide in rat liver. Biochem J 266:363-369

20. Ghidoni R, Sonnino S, Masserini M, Orlando P, Tettamanti G 1981 Specific tritium labeling of gangliosides at the 3-position of sphingosines. J Lipid Res 22:1286-1295

21. Lowry O, Rosebrough N, Farr A, Randall R 1951 Protein measurement with the Folin phenol reagent. J Biol Chem 193:265-275

22. Tettamanti G, Bonali F, Marchesini S, Zambotti V 1973 A new procedure for the extraction, purification and fractionation of brain gangliosides. Biochim Biophys Acta 296:160-170

23. Stahl E 1962 Dunnschicht Chromatographie. Springer Verlag, Berlin, p 498

24. Merril AH Jr, van Echten G, Wang E, Sandhoff K 1993 Fumonisin B1 inhibit sphingosine(sphinganine) $\mathrm{N}$-acyltransferase and de novo sphingolipid biosynthesis in cultured neurons in situ. J Biol Chem 268:27299-27306

25. Rush JS, Panneerselvam K, Waetcher CJ, Freeze HH 2000 Mannose supplementation corrects GDP-mannose deficiency in cultured fibroblasts from some patients with Congenital Disorders of Glycosylation (CDG). Glycobiology 10:829-835

26. Houri JJ, Falbo A, Vignali G, Codogno P, Ghidoni R 1994 The metabolic processing of glycosphingolipids in HT-29 cells is differentiation-dependent. Biochem Biophys Res Commun 202:992-1000

27. Sakiyama H, Takahashi T, Hirabayashi Y, Taniguchi M 1987 Change in the topographical distribution of GM3 during cell spreading and growth: immunostaining with monoclonal antibody against GM3. Cell Struct Funct 12:93-105

28. Berenson CS, Smith TJ 1995 Human orbital fibroblasts in culture express ganglioside profiles distinct from those in dermal fibroblasts. J Clin Endocrinol Metab 80:2668 2674

29. Chigorno V, Tettamanti G, Sonnino S 1996 Metabolic processing of gangliosides by normal and Salla human fibroblasts in culture. A study performed by administering radioactive GM3 ganglioside. J Biol Chem 271:21738-21744

30. Dawson G, Matalon R, Dorfman A 1972 Glycosphingolipids in cultured human skin fibroblasts. I. Characterization and metabolism in normal fibroblasts. J Biol Chem 247:5944-5950

31. Dawson G, Matalon R, Dorfman A 1972 Glycosphingolipids in cultured human skin fibroblasts. II. Characterization and metabolism in fibroblasts from patients with inborn errors of glycosphingolipid and mucopolysaccharide metabolism. J Biol Chem 247:5951-5958

32. Bergmann M, Gross HJ, Abdelatty F, Moller P, Jaeken J, Schwartz-Albiez R 1998 Abnormal surface expression of sialoglycans on B lymphocyte cell lines from patients with carbohydrate deficient glycoprotein syndrome I A (CDGS I A). Glycobiology 8:963-972

33. Marquardt T, Ullrich K, Zimmer P, Hasilik A, Deufel T, Harms E 1995 Carbohydrate-deficient glycoprotein syndrome (CDGS) - glycosylation, folding and intracellular transport of newly synthesized glycoproteins. Eur J Cell Biol 66:268-273

34. Powell LD, Paneerselvam K, Vij R, Diaz S, Manzi A, Buist N, Freeze H, Varki A 1994 Carbohydrate-deficient glycoprotein syndrome: not an N-linked oligosaccharide processing defect, but an abnormality in lipid-linked oligosaccharide biosynthesis? J Clin Invest 94:1901-1909

35. Barone R, Carchon H, Jansen E, Pavone L, Fiumara A, Bosshard NU, Gitzelmann R, Jaeken J 1998 Lysosomal enzyme activities in serum and leukocytes from patients with carbohydrate-deficient glycoprotein syndrome type IA (phosphomannomutase deficiency). J Inherit Metab Dis 21:167-172 